\title{
The importance of antiangiogenic effect in multiple myeloma treatment
}

\author{
Agnieszka Barchnicka”, B-F, Małgorzata Olejniczak-Nowakowska ${ }^{2, E, F}$, \\ Karolina Krupa-Kotara ${ }^{2, E, F}$, Sebastian Grosicki ${ }^{2}$, C, E, F \\ ${ }^{1}$ Department of Doctoral Studies, School of Public Health in Bytom, Medical University of Silesia in Katowice, Poland \\ 2 Department of Cancer Prevention, School of Public Health in Bytom, Medical University of Silesia in Katowice, Poland \\ A - research concept and design; B - collection and/or assembly of data; C - data analysis and interpretation; \\ $D$ - writing the article; $E$ - critical revision of the article; $F$ - final approval of the article
}

Address for correspondence

Agnieszka Barchnicka

E-mail:agnieszka@barchnicka.pl

Funding sources

None declared

Conflict of interest

None declared

Received on 0ctober 24, 2016

Reviewed on November 22, 2016

Accepted on February 7, 2017

DOI

$10.17219 /$ acem/68826

\section{Copyright}

Copyright by Author(s)

This is an article distributed under the terms of the

Creative Commons Attribution Non-Commercial License

(http://creativecommons.org/licenses/by-nc-nd/4.0/)

\begin{abstract}
Angiogenesis plays a significant role in oncogenesis, and thus it has become an attractive target for cancer treatment. It is the formation of new blood vessels that occurs physiologically as well as under pathological conditions, and may influence cancer proliferation and survival. The current therapeutic approach in oncology includes conventional chemotherapy in combination with biologically-based treatment in various perspectives, targeting not only the malignant cells, but also its microenvironment. Target treatment might be less toxic than conventional chemotherapy. In multiple myeloma, there is a close connection between bone marrow stroma, myeloma cell growth and their ability to survive. It has been reported in many clinical observations that the more advanced the multiple myeloma, the more increased the angiogenesis, and this might correlate with the treatment response. There are several angiogenesis inhibitors already registered or in clinical trials in cancer treatment. Despite the continuous research on the development of prognostic factors and introduction of new agents in the treatment, multiple myeloma still remains an incurable and debilitating disease. Some antiangiogenic agents have already been introduced in multiple myeloma treatment, but there is still a need to search for new antiangiogenic drugs and the exploitation of angiogenesis in a clinical approach.
\end{abstract}

Key words: multiple myeloma, angiogenesis, treatment, angiogenesis inhibitors 


\section{Introduction}

Angiogenesis is the formation of new blood vessels, occurring physiologically in various stages of human life. Under pathological conditions, it plays a significant role in the inflammation process, and proliferation and metastasis of most malignant neoplasms. Recent studies have shown a role of angiogenesis and angiogenic factors in the development and course of hematological malignancies. ${ }^{1}$

Multiple myeloma (MM) is a hematological disorder, derived from B lymphocytes that are in the final stage of differentiation. This accounts for approx. 10\% of hematologic malignancies. The essence of multiple myeloma is clonal proliferation of plasma cells in the bone marrow, monoclonal protein production and release of cytokines responsible for the destruction of bone tissue. Symptoms of multiple myeloma depend mainly on the stage of the disease. ${ }^{2}$ Despite the continuous research on the development of prognostic factors and introduction of new agents in the treatment, multiple myeloma still remains an incurable and debilitating disease. ${ }^{3}$

The field of neovascularization was first evaluated in solid tumors. In 1997, Folkman et al. observed an increased amount of microvessels in the bone marrow of children with acute lymphoblastic leukemia. ${ }^{4}$ Angiogenesis is a multifactorial process, associated with the formation of new blood vessels on the basis of the existing vascular network. ${ }^{5}$ It begins with an increase of permeability and widening the lumen of blood vessels. Subsequently, endothelial sprouts are formed, and this process is regulated by several proangiogenic and antiangiogenic factors. ${ }^{6,7}$ In fact, the process of pathologic angiogenesis associated with cancer activity differs from physiological blood vessel formation. The new blood vessel network is chaotic and might be associated with blood flow alteration and increased permeability of the vessels. The proangiogenic growth factors are produced mainly by endothelial cells, but also mast cells and pericytes. In the case of cancer, the "angiogenic switch" is induced. Cancer cells have the potential to produce proangiogenic cytokines, but also to stimulate the environment. Under normal conditions, there is a balance between proangiogenic and antiangiogenic factors. When it is switched, there is an increased angiogenesis potential. Several factors associated with angiogenesis may provide autocrine and paracrine effects to cancer cells. Importantly, the "angiogenic switch" may occur at any stage of cancer, but it is dependent on the cancer type and its interaction with the microenvironment. ${ }^{8}$

\section{Angiogenesis in multiple myeloma}

In multiple myeloma, inducement of proangiogenic and proinflammatory cytokine production is associated with the interaction of myeloma cells and the bone marrow stroma that includes fibroblasts, stromal cells, and also osteoblasts and osteoclasts, monocytes, macrophages, mast cells, and T lymphocytes. In general, in MM there is a tight connection between bone marrow stroma, myeloma cell growth and their ability to survive. The MM cells directly produce some proangiogenic molecules, such as vascular endothelial growth factor (VEGF), basic fibroblast growth factor (bFGF), hepatocyte growth factor (HGF), interleukin-8 (IL-8), osteopontin (OPN), metalloproteinases and angiopoetin-1 (Ang-1), but can also stimulate other cells of the bone marrow microenvironment to secrete different growth factors, and finally endothelial cells to originate the angiogenic process. ${ }^{5}$ The involvement of selected genes associated with angiogenesis in MM disease is described in Table 1.

The increased angiogenesis in MM was first described by Vacca et al., who conducted in vitro research and revealed increased activity of isolated plasma cells from active myeloma patient cells to produce the proangiogenic factors, in comparison with plasma cells derived from patients with monoclonal gammopathy of undetermined significance (MGUS) or inactive MM. ${ }^{9}$ This observation was followed by continuous research that showed increased bone marrow

Table 1. Involvement of selected genes and proteins associated with angiogenesis in MM disease $\mathrm{S}^{5,16,17,19}$

\begin{tabular}{|l|c|c|}
\hline \multicolumn{1}{|c|}{ Gene and protein name } & Impact on angiogenesis & Expression by MM cells \\
\hline VEGF & proangiogenic & expressed \\
\hline HGF & proangiogenic & aberrantly expressed \\
\hline ANG & proangiogenic & aberrantly expressed \\
\hline IL-8 & proangiogenic & upregulated (vs normal plasma cells) \\
\hline ANGPT1 & proangiogenic & expressed \\
\hline MMP-9 & proangiogenic & expressed (potential prognosis factor) \\
\hline TSP-1 & antiangiogenic & aberrantly expressed \\
\hline ADAMTS9 & antiangiogenic & downregulated (vs normal plasma cells) \\
\hline LAMA5 & antiangiogenic & plasma cells) \\
\hline
\end{tabular}

VEGF - vascular endothelial growth factor; HGF - hepatocyte growth factor; ANG - angiogenin; IL-8 - interleukin-8; ANGPT1 - angiopoetin-1; MMP-9 - matrix metalloproteinase-9; TSP-1 - thrombospondin-1; ADAMTS9 - a disintegrin and metalloproteinase with throbospondin motifs 9; LAMA5 - laminin alpha 5. 


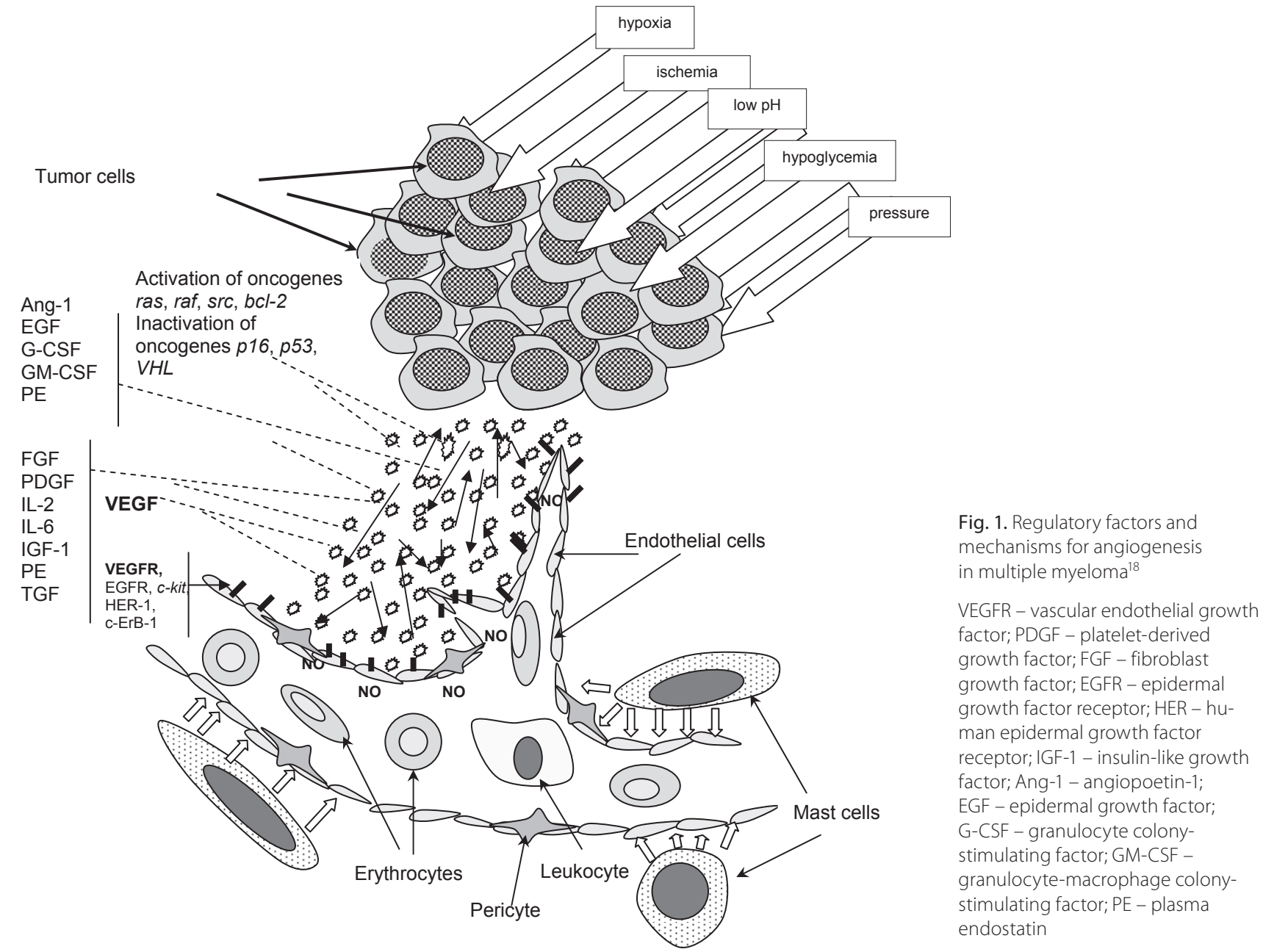

angiogenesis in patients with advanced MM. It has been revealed that bone marrow microvessel density (MVD) assessed in histopathological samples was substantially higher in these patients than in health controls. In a study by Rajkumar et al., angiogenesis was assessed in bone marrow from 400 patients with multiple myeloma, MGUS and primary amyloidosis. It was found that the degree of angiogenesis is lower in patients with MGUS, and significantly increased in patients with multiple myeloma. ${ }^{10}$ The greater intensity of blood vessel formation, the higher the proliferation of plasma cells seems to be, so it could indicate that angiogenesis might be associated with progression of the disease. ${ }^{10}$ The assessment of bone marrow MVD might be an independent prognostic factor at the moment of diagnosis as well as in the response assessment. ${ }^{11-13}$

Several studies have been conducted on the relationship between the levels of angiogenic factors in the blood and MVD. The increased levels of proangiogenic molecules might also be associated with the treatment and prognosis. ${ }^{14,15}$ The proangiogenic factors differ in respect of the activity. VEGF-A has the highest proangiogenic potential, and it is the main factor in angiogenesis and vasculogenesis. When the release of VEGF-A is inhibited, a regression of existing and formation of new blood vessels might be observed. A study conducted by Mileshkin et al. has shown that VEGF levels decreased significantly in MM patients who responded to thalidomide therapy. ${ }^{16}$ On the other hand, the research by Cibeira et al. has not confirmed any correlation between VEGF level and response rate. ${ }^{17}$

Apart from releasing and stimulating angiogenic factors, the MM cells might also influence new blood vessel formation by direct interaction with matrix cells and fibronectin (Fig. 1). ${ }^{18}$ Endothelial cells located in the bone marrow microenvironment may also produce angiogenic factors that act in a dual way (autocrine and paracrine) on both MM and endothelial cells. Thus, there is a mutual stimulation between endothelial cells and malignant plasma cells. ${ }^{19}$

\section{Angiogenesis inhibitors in cancer treatment}

The current therapeutic approach in oncology includes conventional chemotherapy in combination with biologically-based treatment in various perspectives, targeting not only the malignant cells but also its microenvironment, so new therapeutic targets are available nowadays. Angiogenesis seems to be a key factor in malignant tumor growth and survival, so there are some antiangiogenic agents already registered and others being investigated in 
Table 2. Selected angiogenesis inhibitors in cancer treatment ${ }^{19}$

\begin{tabular}{|c|c|c|c|}
\hline Drug & Signaling pathway & Molecular target & Indication (registered or in clinical trial) \\
\hline Thalidomide & RTKs & VEGFR & multiple myeloma \\
\hline Bevacizumab & RTKs & VEGFR-A & $\begin{array}{l}\text { non-small lung cancer } \\
\text { breast cancer } \\
\text { glioblastoma } \\
\text { colorectal cancer } \\
\text { renal cell carcinoma }\end{array}$ \\
\hline Aflibercept & RTKs & VEGFR-1 and -2 & \\
\hline Sunitinib & RTKs & $\begin{array}{l}\text { VEGFR } \\
\text { PDGFR } \\
\text { c-kit } \\
\text { FLT-3 } \\
\text { CSF-1R }\end{array}$ & $\begin{array}{l}\text { gist } \\
\text { renal cell carcinoma } \\
\text { breast cancer }\end{array}$ \\
\hline Crizotinib & RTKs & $\begin{array}{l}\text { c-Met } \\
\text { HGFR }\end{array}$ & $\begin{array}{l}\text { lung cancer } \\
\text { breast cancer }\end{array}$ \\
\hline Sofarenib & RTKs & $\begin{array}{c}\text { Raf-kinase (B-Raf, C-Raf) } \\
\text { VEGFR-2 and -3 } \\
\text { PDGFR- } \beta \\
\text { c-kit }\end{array}$ & $\begin{array}{l}\text { colon cancer } \\
\text { pancreatic cancer } \\
\text { breast cancer }\end{array}$ \\
\hline Semaxanib & RTKs & VEGFR & $\begin{array}{c}\text { colorectal cancer } \\
\text { (clinical development stopped due to severe } \\
\text { thromboembolic adverse events) }\end{array}$ \\
\hline Erlotinib & RTKs & EGFR/HER-1 & $\begin{array}{c}\mathrm{nscl} \\
\text { pancreatic cancer }\end{array}$ \\
\hline Imatinib & RTKs & $\begin{array}{l}\text { PDGFR } \\
c-k i t\end{array}$ & antiangiogenic inhibition in vitro \\
\hline
\end{tabular}

RTKs - receptor tyrosine kinases; VEGFR - vascular endothelial growth factor; PDGFR - Platelet-derived growth factor receptors; HGFR - hepatocyte growth factor receptor; CSF-1R - Colony stimulating factor 1 receptor; EGFR - epidermal growth factor receptor; HER - human epidermal growth factor receptor.

clinical trials. It has been reported in animal models that target therapy might be less toxic than conventional chemotherapy. Angiogenesis is a complex pathway, involving a number of factors, so there is a possibility to inhibit it on different levels. ${ }^{18}$ It is crucial to distinguish between agents that impact angiogenesis and vascular targets. Antiangiogenic drugs mainly affect new vessel growth and formation in cancer tissue. Vascular targeting agents may destroy the existing vasculature of the tumor. The actions of both kinds of agents may synergize. There are several molecules of interest as potential therapeutic targets that have a regulatory or signaling function involved in angiogenesis, such as growth factors (e.g., VEGF, fibroblast growth factor - FGF, epidermal growth factor - EGF), transcription factors like hypoxia-inducible factor (HIF), and receptor tyrosine kinases. The same applies to molecules involved in phosphatidylinositol-4,5-biosphosphate-3-kinase (PI3K) and mitogen-activated protein kinase (MAPK) signaling.

Bevacizumab is the humanized monoclonal antibody VEGF, and is approved in combination with chemotherapy for the treatment of some cancers (non-small lung cancer, breast cancer, glioblastoma, colorectal cancer, and renal cell carcinoma). This acts by inhibition of VEGF receptors, thereby also interfering with the autocrine and paracrine mechanisms of malignant cell survival mediated by VEGFR-1 and VEGFR-2. Bevacizumab is also in clinical trials in metastatic breast cancer and colorectal cancer. ${ }^{20}$
The most important agents with antiangiogenic potential in clinical trials or already registered in cancer treatment are presented in Table 2.

\section{Angiogenesis inhibitors in MM}

As angiogenesis seems to be strongly associated with MM development and prognosis, there have been increasing efforts to search for treatment with antiangiogenic potential. There are some drugs already registered in MM treatment that have demonstrated an antiangiogenic effect. These drugs might be administered alone or along with chemotherapy.

\section{Immunomodulatory drugs}

Thalidomide was the first agent introduced to MM treatment because of its potential antiangiogenic action. The drug was synthetized in the early 1950s and prescribed because of its sedative effect; finally it was withdrawn due to serious teratogenic side effects. In the 1990s, a study of rabbit model corneal neovascularization induced in response to bFGF demonstrated the antiangiogenic activity of thalidomide. ${ }^{21}$ Afterwards, the clinical efficacy of thalidomide in MM patients was reported. Singhal et al. 
showed a response to thalidomide monotherapy in a clinical phase II trial that included previously treated patients with refractory disease. ${ }^{22}$ Partial response was achieved in almost $1 / 3$ of patients and $14 \%$ achieved a complete or nearly complete remission. ${ }^{22}$ That was the beginning of subsequent clinical studies and, in consequence, thalidomide was approved by the United States Federal Drug Administration (FDA) for the therapy of newly-diagnosed MM patients in combination with dexamethasone. The results of a phase III trial showed that the response rate in patients treated with thalidomide and dexamethasone was $63 \%$ in comparison to $41 \%$ in patients treated with dexamethasone in monotherapy. ${ }^{23}$ The mechanism of the antiangiogenic effect associated with thalidomide is actually unknown. It has been observed that thalidomide might have important immunomodulatory effects associated with a decreasing synthesis of TNF- $\alpha$ and shifting the $\mathrm{T}$ cell population toward $\mathrm{T}$ helper. Thalidomide also decreases the expression of intercellular and vascular cell adhesion molecules (intercellular adhesion molecule 1 ICAM-1 and vascular cell adhesion molecule - VCAM), and thus attenuates the interaction between stromal and malignant plasma cells. ${ }^{24}$ In a study by Gupta et al., thalidomide and the newer immunomodulatory drugs like lenalidomide have been reported to significantly decrease the expression of proangiogenic factors, mainly VEGF and interleukin-6 (IL-6), in MM patients. ${ }^{25}$ However, as has been reported in some other studies, the thalidomide action seems not always to be associated with a decrease of bone marrow angiogenesis and the levels of cytokines related to angiogenesis. ${ }^{17}$ Moreover, in spite of high vascularity of extramedullary plasmocytoma, it has been observed that it does not respond to thalidomide treatment. ${ }^{26}$

Lenalidomide is derived from a modified thalidomide chemical structure and it also shows the immunomodulatory effects with lower rates of adverse events. In patients that were previously treated and had relapsed or refractory MM, lenalidomide in combination with dexamethasone showed a significant increase in the response rate, from $22.5 \%$ to $59.2 \%$, in comparison to dexamethasone alone. ${ }^{27}$ Lenalidomide was previously approved by the FDA for second-line therapy in MM and, further, in 2015 for newly diagnosed patients. The mechanisms of action mainly seen in MM may comprise induction of cell cycle arrest by an increase in expression of the cyclin-dependent kinase inhibitor p21 and decrease in expression of interferon regulatory factor 4 , and also induction of apoptosis and attenuation of angiogenesis. ${ }^{28}$

$\mathrm{Lu}$ et al. conducted in vitro research and reported that lenalidomide may inhibit the formation of microvessels in a dose-dependent manner. ${ }^{29}$ The inhibitory effect of lenalidomide may derive from the associations between cadherin 5, $\beta$-catenin and CD31. Moreover, lenalidomide was shown to attenuate PI3K-Akt pathway signaling induced by VEGF, which is known to modulate adherence junction formation. ${ }^{29}$ In a Dredge et al.'s study, it was observed that le antiangiogenic effect of lenalidomide might not be related to a decrease of endothelial cell proliferation, still the migration of endothelial cells is inhibited significantly. ${ }^{30}$ The study has been extended and it has been reported that lenalidomide may inhibit the action of some proangiogenic molecules in an animal model. ${ }^{31}$

Pomalidomide is a novel anti-myeloma agent among the immunomodulatory class drugs. Preclinical studies have shown that pomalidomide is active against MM cell lines in cases of bortezomib and lenalidomide resistance, which has been confirmed in a number of further clinical trials with a relatively tolerable safety profile. ${ }^{32,33}$ Aside from these mechanisms of actions, pomalidomide inhibits stromal cell adhesion in bone marrow and has been shown to significantly inhibit angiogenesis by targeting VEGF and hypoxia-inducible factor- $1 \alpha$ (HIF1- $\alpha$ ), a transcription factor regulating angiogenesis by induction of VEGF transcription. ${ }^{34}$

\section{Proteasome inhibitors}

Bortezomib, a modified boronic acid dipeptide, is a selective inhibitor of nuclear factor- $\mathrm{K} B(\mathrm{NFK} B)$ and has been approved for clinical use for the treatment of MM patients, but also shows activity in other hematological malignancies. It blocks very specifically the $ß$-subunit of the $26 \mathrm{~S}$ proteasome. ${ }^{35}$ The anticancer activity of bortezomib might also be associated with angiogenesis inhibition. It has been previously reported that proteasome inhibitors have antiangiogenic potential in animal models. ${ }^{36}$ Inhibition of NFKB mediated by bortezomib is supposed to be involved in targeting HIF-1 $\alpha$-mediated VEGF expression. ${ }^{37}$ Roccaro et al. examined the activity of bortezomib on the angiogenic phenotype of multiple myeloma patient-derived endothelial cells (MMEC). The authors reported that bortezomib induced a dose-dependent inhibition of VEGF and IL- 6 production, and confirmed reverse transcriptase-PCR related to drug downregulation, IL-6, insulin-like growth factor-I (IGF-1), angiopoietin 1 (Ang1), and angiopoietin 2 (Ang2) transcription. ${ }^{38}$

Carfilzomib is a second-generation proteasome inhibitor, which has been approved by the FDA for clinical use in relapsed or refractory MM patients. In preclinical trials, carfilzomib demonstrated more potent antimyeloma activity than bortezomib. ${ }^{39}$ It has got a similar antiangiogenic potential like bortezomib.

\section{Agents targeting VEGF}

In the study by White et al. (AMBER trial), bevacizumab was administered in combination with bortezomib for the treatment of relapsed/refractory MM patients. There was no significant increase of progression free survival (PFS) when bevacizumab was given along with bortezomib vs 
bortezomib in monotherapy. ${ }^{40}$ Similar unsatisfactory observations have been reported about other VEGF receptor-targeted agents, such as semaxanib (SU5416), zactima (ZD6474), and pazopanib (GW786034). ${ }^{41-43}$ Although antiVEGF antibodies might suppress a pathway involved in malignant myeloma cell growth, there are other factors associated with the disease activity and progression that these agents might not add any clinical benefits to in the treatment.

\section{Novel antiangiogenic strategies}

It has been suggested that modifications in multicellular eukaryotic messenger RNA (miRNA) expression may participate in the pathogenesis of most cancers in humans. ${ }^{44}$ MicroRNAs are actually the class of non-coding RNAs (22-nucleotide) that operate in RNA silencing and posttranscriptional regulation of gene expression. They maintain biological events in various settings, such as cell growth, differentiation and apoptosis, but also metabolism of fat and viral infection. It has been observed that some miRNAs may be involved in controlling the production of angiogenic molecules, and therefore the angiogenesis process. Roccaro et al. have demonstrated that miR-15a and miR-16 are significantly diminished or lacking in relapsed/refractory MM patients. In vitro, as the regulators of MM pathogenesis, miR-15a and miR-16 attenuate the formation of capillaries and MM cell-associated endothelial cell growth. When premiR-15a and pre-miR-16 were transfected into malignant plasma cells, this contributed to significant inhibition of VEGF secretion. The authors have concluded that the field of regulation of VEGF by these miRNAs in MM patients' needs to be investigated. ${ }^{45}$ Furthermore, Sun et al. conducted a study concerning the miR-15a and miR-16 expression levels and their association with the advanced stage of MM patients. The miRNAs were found to be downregulated in malignant plasma cells, and a correlation between downregulation and the MM stage was established. The results also confirmed the previous observations that miR-15a and miR-16 expression may inhibit the proangiogenic activity of malignant plasma cells. ${ }^{46}$ Currently, there have been several reports that hypoxia is involved in miRNA expression in cancer. Another miRNA that is downregulated in MM is miR-199a-5p, and this has become an important issue of concern, as it directly targets HIF1- $\alpha$, a transcription factor regulating angiogenesis, by induction of VEGF transcription. ${ }^{47}$ A Raimondi et al.'s study has revealed that enforced expression of miR-199a-5p contributed to downregulation of HIF- $1 \alpha$ expression and, moreover, some other proangiogenic factors such as FGFb, VEGF-A and IL-8 in hypoxic malignant plasma cells in vitro. ${ }^{48}$

\section{Summary}

Multiple myeloma is a unique hematological cancer wherein abnormal plasma cells, through interaction, subordinate the microenvironment for their own growth and expansion. MM might be a metastatic as well as a localized disease. Every single time it remodels the environment, destroying the bone structure and forcing angiogenesis, and thus provides a supply of substances essential to its survival. Awareness of these mechanisms may enable the development of a strategy for prolonged treatment based on specific inhibitors that could stabilize the treatment effect, and thus extend patients' survival. Research on the regulatory mechanisms of angiogenesis in MM according to their complexity and usefulness for treatment should be continued.

\section{References}

1. Carmeliet P. Angiogenesis in health and disease. Nat Med. 2003;9: 653-660.

2. Rajkumar SV. Myeloma today: Disease definitions and treatment advances. Am J Hematol. 2016;91(1): 90-100.

3. Rajkumar S, Kumar S. Multiple myeloma: Diagnosis and treatment. Mayo Clin Proc. 2016;91(1):101-119.

4. Folkman J. Seminars in medicine of the Beth Israel Hospital, Boston. Clinical applications of research on angiogenesis. N Engl J Med. 1995;333(26):1757-1763.

5. Otjacques E. Biological aspects of angiogenesis in multiple myeloma. Int J Hematol. 2011;94:505-518.

6. Cines DB, Pollak ES, Buck CA, et al. Endothelial cells in physiology and in the pathophysiology of vascular disorders. Blood. 1998;91(10): 3527-3561.

7. De Bock K, Georgiadou M, Carmeliet P. Role of endothelial cell metabolism in vessel sprouting. Cell Metab. 2013;18(5):634-647.

8. Bergers G, Benjamin LE. Tumorigenesis and the angiogenic switch. Nat Rev Cancer. 2003;3:401-410.

9. Vacca A, Ribatti D, Presta M, et al. Bone marrow neovascularization, plasma cell angiogenic potential, and matrix metalloproteinase-2 secretion parallel progression of human multiple myeloma. Blood. 1999;93:3064-3073.

10. Rajkumar VS, Mesa RA, Fonseca R, et al. Bone marrow angiogenesis in 400 patients with monoclonal gammopathy of undetermined significance, multiple myeloma, and primary amyloidosis. Clin Cancer Res. 2002; 8(7):2210-2216.

11. Sezer $\mathrm{O}$, Niemöller K, Eucker J, et al. Bone marrow microvessel density is a prognostic factor for survival in patients with multiple myeloma. Ann Hematol. 2000;79(10):574-577.

12. Sezer O, Niemöller K, Jakob C, et al. Relationship between bone marrow angiogenesis and plasma cell infiltration and serum beta2-microglobulin levels in patients with multiple myeloma. Ann Hematol. 2001;80(10):598-601.

13. Lee $\mathrm{N}$, Lee $\mathrm{H}$, Moon SY, et al. Adverse prognostic impact of bone marrow microvessel density in multiple myeloma. Ann Lab Med. 2015; 35(6):563-569.

14. Sezer O, Jakob C, Eucker J, et al. Serum levels of the angiogenic cytokines basic fibroblast growth factor (bFGF), vascular endothelial growth factor (VEGF) and hepatocyte growth factor (HGF) in multiple myeloma. Eur J Haematol. 2001;66:83-88.

15. Kokonozaki M, Tsirakis G, Devetzoglou M, et al. Potential role of FLT-3 ligand in the angiogenic process of multiple myeloma. Leuk Res 2015;39:1467-1472.

16. Mileshkin L, Honemann D, Gambell P, et al. Patients with multiple myeloma treated with thalidomide: Evaluation of clinical parameters, cytokines, angiogenic markers, mast cells and marrow CD57+ cytotoxic T cells as predictors of outcome. Haematologica. 2007; 92(8):1075-1082. 
17. Cibeira MT, Rozman M, Segarra M, et al. Bone marrow angiogenesis and angiogenic factors in multiple myeloma treated with novel agents. Cytokine. 2008;41(3):244-253.

18. Grosicki S, Grosicka A, Hołowiecki J. Kliniczne znaczenie angiogenezy i czynników ją modyfikujących w onkohematologii. Wiad Lek. 2007;60(1/2):39-46.

19. Cook K, Figg W. Angiogenesis inhibitors: Current strategies and future prospects. CA Cancer J Clin. 2010;60(4):222-243.

20. Pour L, Svachova $H$, Adam Z, et al. Levels of angiogenic factors in patients with multiple myeloma correlate with treatment response. Ann Hematol. 2010;89:385.

21. D'Amato RJ, Loughnan MS, Flynn E, Folkman J. Thalidomide is an inhibitor of angiogenesis. Proc Natl Acad SciUSA. 1994;91:4082-4085.

22. Singhal S, Mehta J, Desikan R, et al. Antitumor activity of thalidomide in refractory multiple myeloma. N Engl J Med. 1999;341:1565-1571.

23. Rajkumar SV, Blood E, Vesole D, Fonseca R, Greipp PR; Eastern Cooperative Oncology Group. Phase III clinical trial of thalidomide plus dexamethasone compared with dexamethasone alone in newly diagnosed multiple myeloma: A clinical trial coordinated by the Eastern Cooperative Oncology Group. J Clin Oncol. 2006;24(3):431-436.

24. Dmoszyńska A. Angiogeneza i leczenie antyangiogenne w szpiCzaku plazmocytowym. Onkol Prak Klin. 2009;5 (Suppl A):A56-A61.

25. Gupta D, Treon SP, Shima Y, et al. Adherence of multiple myeloma cells to bone marrow stromal cells upregulates vascular endothelial growth factor secretion: Therapeutic applications. Leukemia. 2001;15:1950-1961.

26. Rosiñol L, Cibeira MT, Blade J, et al. Extramedullary multiple myeloma escapes the effect of thalidomide. Haematologica. 2004;89:832-836.

27. Dimopoulos $M$, Spencer $A$, Attal $M$, et al. Lenalidomide plus dexamethasone for relapsed or refractory multiple myeloma. $N$ Engl J Med. 2007;357:2123-2132.

28. Guirguis AA, Ebert BL. Lenalidomide: Deciphering mechanisms of action in myeloma, myelodysplastic syndrome and beyond. Curr Opin Cell Biol. 2015;37:61-67.

29. Lu L, Payvandi F, Wu L, et al. The anti-cancer drug lenalidomide inhibits angiogenesis and metastasis via multiple inhibitory effects on endothelial cell function in normoxic and hypoxic conditions. Microvasc Res. 2009;77:78-86.

30. Dredge K, Marriott JB, Macdonald CD, et al. Novel thalidomide analogues display anti-angiogenic activity independently of immunomodulatory effects. Br J Cancer. 2002;87(10):1166-1172.

31. Dredge $K$, Horsfall R, Robinson SP, et al. Orally administered lenalidomide (CC-5013) is anti-angiogenic in vivo and inhibits endothelial cell migration and Akt phosphorylation in vitro. Microvasc Res. 2005;69(1-2):56-63.

32. Lacy MQ, Hayman SR, Gertz MA, et al. Pomalidomide (CC4047) plus low-dose dexamethasone as therapy for relapsed multiple myeloma. J Clin Oncol. 2009;27(30):5008-5014.

33. Leleu X, Attal M, Arnulf B, et al. Pomalidomide plus low-dose dexamethasone is active and well tolerated in bortezomib and lenalid- omide-refractory multiple myeloma: Intergroupe Francophone du Myelome 2009-02. Blood. 2013;121(11):1968-1975.

34. Chanan-Khan AA, Swaika A, Paulus A, et al. Pomalidomide: The new immunomodulatory agent for the treatment of multiple myeloma. Blood Cancer J. 2013;3(9):e143.

35. Grosicki S, Barchnicka A, Jurczyszyn A, Grosicka A. Bortezomib for the treatment of multiple myeloma. Expert Rev Hematol. 2014;7(2):173-185.

36. Sunwoo JB, Chen Z, Dong G, et al. Novel proteasome inhibitor PS-341 inhibits activation of nuclear factor-kappa B, cell survival, tumor growth, and angiogenesis in squamous cell carcinoma. Clin Cancer Res. 2001;7:1419-1428.

37. McConkey DJ, Zhu K. Mechanisms of proteasome inhibitor action and resistance in cancer. Drug Resist Updat. 2008;11(4-5):164-179.

38. Roccaro AM, Hideshima T, Raje N, et al. Bortezomib mediates antiangiogenesis in multiple myeloma via direct and indirect effects on endothelial cells. Cancer Res. 2006;66(1):184-191.

39. Kuhn DJ, Chen Q, Voorhees PM, et al. Potent activity of carfilzomib, a novel, irreversible inhibitor of the ubiquitin-proteasome pathway, against preclinical models of multiple myeloma. Blood. 2007;110:3281-3290.

40. White D, Kassim A, Bhaskar B, Yi J, Wamstad K, Paton VE. Results from AMBER, a randomized phase 2 study of bevacizumab and bortezomib versus bortezomib in relapsed or refractory multiple myeloma. Cancer. 2013;119(2):339-347.

41. Zangari M, Anaissie E, Stopeck A, et al. Phase II study of SU5416, a small molecule vascular endothelial growth factor tyrosine kinase receptor inhibitor, in patients with refractory multiple myeloma. Clin Cancer Res. 2004;10(1 Pt 1):88-95.

42. Kovacs MJ, Reece DE, Marcellus D, et al. A phase II study of ZD6474 (Zactima, a selective inhibitor of VEGFR and EGFR tyrosine kinase) in patients with relapsed multiple myeloma - NCIC CTG IND.145. Invest New Drugs. 2006;24:529-535.

43. Prince HM, Honemann D, Spencer A, et al. Vascular endothelial growth factor inhibition is not an effective therapeutic strategy for relapsed or refractory multiple myeloma: A phase 2 study of pazopanib (GW786034). Blood. 2009;113:4819-4820.

44. Calin GA, Croce CM. MicroRNA-cancer connection: The beginning of a new tale. Cancer Res. 2006;66:7390-7394.

45. Roccaro AM, Sacco A, Thompson B, et al. MicroRNAs 15a and 16 regulate tumor proliferation in multiple myeloma. Blood. 2009;113:66696680.

46. Sun $C Y$, She $X M$, Qin $Y$, et al. miR-15a and miR-16 affect the angiogenesis of multiple myeloma by targeting VEGF. Carcinogenesis. 2013;34(2):426-423.

47. Gu S, Chan WY. Flexible and versatile as a chameleon: Sophisticated functions of microRNA-199a. Int J Mol Sci. 2012;13(7):84498466.

48. Raimondi L, Amodio N, Di Martino MT, et al. Targeting of multiple myeloma-related angiogenesis by miR-199a-5p mimics: In vitro and in vivo anti-tumor activity. Oncotarget. 2014;5(10):3039-3054. 